\title{
Seizures and Failures in the Giant Fiber Pathway of Drosophila Bang-Sensitive Paralytic Mutants
}

\author{
Paul Pavlidis ${ }^{1, a}$ and Mark A. Tanouye ${ }^{1,2}$ \\ 'Department of Molecular and Cell Biology, Division of Neurobiology, University of California, Berkeley, California \\ 94720 and ${ }^{2}$ Department of Environmental Science, Policy and Management, University of California, Berkeley, \\ California 94720
}

\begin{abstract}
Drosophila bang-sensitive paralytic mutants suffer from hyperactivity and paralysis following a mechanical shock; after recovery from paralysis, they cannot be paralyzed for a refractory period lasting up to $1 \mathrm{hr}$. Previously, we have shown that in easily shocked (eas), a typical bang-sensitive mutant, electrical shocks delivered to the brain cause seizure-like activity in the dorsal longitudinal flight motor neurons (DLMmns), and failure of giant fiber (GF) stimulation to evoke DLM potentials via the escape response pathway (Pavlidis et al., 1994). Here, we show that seizure and failure in the GF pathway with a refractory period is common to all six members of the bang-sensitive class. This syndrome was not found in any of eight other excitability mutants, including those affecting voltage-gated sodium or potassium-channel function. We show that failure occurs at the synapse between a peripherally synapsing interneuron (PSI) and the DLMmns, while the DLMmn-DLM neuromuscular junctions remain functional. Additionally, failure occurs in all other GF pathway-activated muscles. Failures occurred without seizures in the tergotrochanteral jump muscle (TTM), as was also found in $\sim \mathbf{1 0} \%$ of DLM tests, suggesting that seizures and failures may be independent events. This hypothesis is supported by the finding that, in double mutant combination with $m / e^{\text {napts }}$, which suppresses behavioral bang sensitivity, DLM failures, but not seizures, were reduced.
\end{abstract}

[Key words: Drosophila melanogaster, paralysis, seizure, synaptic transmission, hyperexcitability, giant fiber pathway]

A genetic analysis of behavioral defects in Drosophila has proven valuable in identifying genes involved in regulating nervous system excitability, such as ion channel genes (Wu and Ganetzky, 1992). It is thought that the knowledge of nervous system function gained by the study of Drosophila mutants can be ap-

\footnotetext{
Received Feb. 2, 1995; revised April 10, 1995; accepted April 13, 1995.

We thank Sally Faulhaber for assistance in maintenance of Drosophila stocks, Elaine R. Reynolds for constructing the bss; mlen apts double mutants and sharing preliminary data on $s d a$, and David Stellwagen for assistance and discussion. Tim Tully and John Palka generously provided iso7.8 (sda) and 2206 flies, respectively. We also thank the members of the Tanouye lab for discussion and comments on the manuscript. Part of this work was conducted under support by a grant from the NIH (N531231-01A2).

Correspondence should be addressed to Mark A. Tanouye, Department of Environmental Science, Policy and Management, University of California, Berkeley, CA 94720.

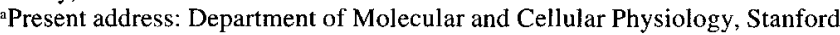
University Medical Center, Stanford, CA 94305.

Copyright (C) 1995 Society for Neuroscience $0270-6474 / 95 / 155810-10 \$ 05.00 / 0$
}

plied to the study of mammalian nervous system function and pathology (Benzer, 1971). An underlying assumption of this approach is that although there are major differences between insect and mammalian nervous systems at the multicellular organizational level, many of the fundamental cellular and molecular mechanisms regulating excitability are conserved.

The present work focuses on a poorly understood class of Drosophila behavioral mutants, the bang-sensitive paralytics, which suffer from intense hyperactivity and temporary paralysis following a mechanical shock, such as a tap of the culture vial on the benchtop or brief vortex mixing (a "bang") (Benzer, 1971; Ganetzky and Wu, 1982a). The hyperactivity, which is a unique feature of this class of mutants, is characterized by intense, uncoordinated motor activity including wing flapping, leg shaking, and abdominal muscle contractions (Benzer, 1971; Pavlidis, 1994; Pavlidis et al., 1994). A comparison of this behavioral defect to a mammalian epileptic seizure has been made (Benzer, 1971), although the hypothesis that the underlying excitability defect in these mutants might have a functional relationship with excitability defects underlying mammalian seizure syndromes has not been explored. More recently, it has been found that the first genetically transmitted mammalian epilepsy for which the responsible gene has been cloned, human myoclonic epilepsy/ragged red fiber (MERRF) disease, is due to a mutation in a mitochondrial lysine tRNA gene (Shoffner et al., 1990), suggesting a similarity with the first bang-sensitive gene to be cloned, technical knockout (tko), which encodes a mitochondrial ribosomal protein (Royden et al., 1987).

In an examination of the giant fiber (GF) escape response pathway (Fig. 1) of the bang-sensitive easily shocked (eas), Pavlidis et al. (1994) described a physiological defect that appears to account for eas behavior, including the hyperactivity. A short, intense train of electrical stimuli (a $100 \mathrm{msec}$ "buzz") delivered to the brain of the fly could mimic the effects of a mechanical bang on eas (Pavlidis et al., 1994). The buzz was followed by intense trains of abnormal, high-frequency $(>100 \mathrm{~Hz})$ dorsal longitudinal muscle (DLM) activity, lasting $1-2 \mathrm{sec}$, and by failure, lasting $\sim 100 \mathrm{sec}$, of the DLM excitatory synaptic response to GF pathway stimulation. These events closely follow the characteristics and time course of the eas behavior (Pavlidis et al., 1994).

The purpose of the present study is threefold. First, we surveyed the bang sensitives and other excitability mutants for the GF pathway defects found in eas. Second, we extended our investigation of this defect to other muscles activated by the GF pathway, and attempted to identify the sites of failure in the 


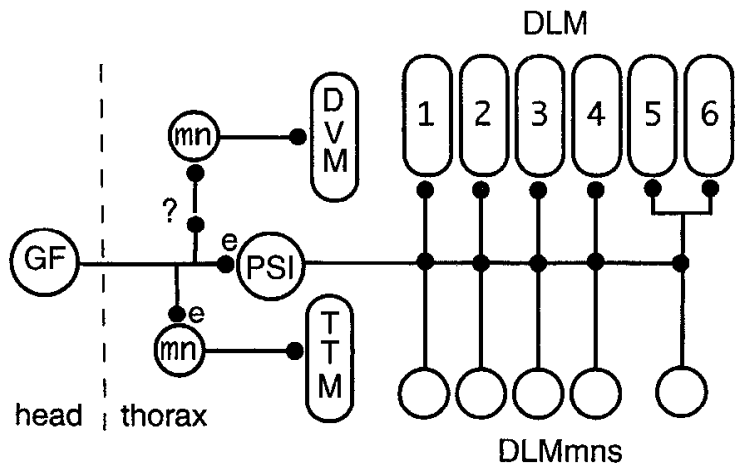

Figure I. The giant fiber pathway. One of two bilaterally symmetric GF pathways is diagrammed schematically. Neurons are depicted as circles and muscles as ovals. All synapses (small black spots) are chemical except those marked $e$ (electrical junctions). The GF is an interneuron that projects from the brain to the thoracic ganglion via the cervical connective. The GF has several outputs in the thorax. First, the GF synapses with the TTMmn, which drives the large tubular TTM. Second, the GF synapses with another interneuron, the peripherally synapsing interneuron $(P S I)$, which drives the DLMmns via axoaxonic chemical synapses. The PSI axon does not branch in making these synapses; rather, the synapses are highly localized at the termination of the PSI axon in the posterior dorsal mesothoracic nerve (PDMN), which contains all five DLM motor neuron axons (King and Wyman, 1980). When the GF is stimulated, all six DLM fibers are activated synchronously via the PSI and DLMmns (Tanouye and Wyman, 1980). Note that DLM fibers 5 and 6 are innervated by a single motor neuron (Ikeda et al., 1980). Third, the GF has an output to the DVMs (only one of six fibers is depicted). This output is not well characterized but is probably includes at least one interneuron (Tanouye and Wyman, 1980). The DLM and DVM motor neurons also receive inputs from other pathways responsible for flight activity (not shown).

pathway. Finally, we examined the relationship between bangsensitive seizures and failures. The results strongly suggest that bang sensitivity is due to a similar physiological defect in all the mutants, giving rise to seizures and synaptic failures. Since there are many genetic loci that can give rise to bang sensitivity, these mutants provide a unique opportunity to dissect complex aspects of neuronal excitability.

\section{Materials and Methods}

Fly stocks. Drosophila melanogaster strains were reared and studied at room temperature $\left(22-24^{\circ} \mathrm{C}\right)$. A complete list of the strains used in this study is provided in Table 1. Wild type flies were the Canton-Special (CS) strain. Behavioral mutants were obtained from several sources, primarily the collections of B. Ganetzky (University of Wisconsin, Madison, WI) and C.-F. Wu (University of Iowa, Iowa City, IA). The P-element insertion 2206 was a gift of J. Palka (University of Washington, Seattle, WA). A newly identified bang-sensitive line, iso7.8, was a gift of T. Tully (Cold Spring Harbor Laboratory, Cold Spring Harbor, NY). The locus defined by iso 7.8 has been named slamdance (sda) and maps to the third chromosome (E. R. Reynolds, personal communication). $s d a^{i s o 7.8}$ has a typical bang-sensitive phenotype (Reynolds, personal communication). eas is the only bang sensitive other than $t k o$, for which the responsible gene has been cloned; eas encodes ethanolamine kinase, which is required for one pathway of ethanolamine phospholipid synthesis (Pavlidis et al., 1994). The other bang-sensitive mutants used in this study were bss, bang sensitive (bas), and knockdown $(k d n)$.

The other, non-bang-sensitive mutants were chosen to represent a cross-section of the types of behavioral and physiological defects found in Drosophila, and which, in particular, had the greatest likelihood of having a relationship to the bang sensitives. Three mutants, Shaker (Sh; a potassium channel gene defect), Hyperkinetic ( $H k$; which probably affects potassium channel function, Stern and Ganetzky, 1989), and Frequenin (Frq; affects a calcium binding protein), were selected because the larval neuromuscular junctions of these mutants rapidly develop large, prolonged postsynaptic potentials following high-frequency nerve stimulation in a manner similar to bss (Jan et al., 1977; Stern and Ganetzky, 1989; Mallart et al., 1991). seizure (seits) was chosen both because of its presumed effect on sodium channels and the observation of severe DLM hyperactivity at restrictive temperatures (Jackson et al., 1985; Kasbekar et al., 1987). shibire (shits) affects a dynamin-like protein required for synaptic vesicle membrane recycling (Kosaka and Ikeda, 1983; van der Bliek and Meyerowitz, 1991), while paralyzed (para ${ }^{i s}$ ), encodes a major voltage-galed sodium channel (Loughney, et al., 1989). 2206 is a P-element mutation in the $\alpha-\mathrm{Na}^{+}-\mathrm{K}^{+}$ATPase gene and displays a weak mechanical shock-sensitive phenotype (Schubiger et al., 1994). maleless-no-action potential (mlentupts) is a temperature-sensitive paralytic mutation affecting a RNA-helicase-like protein and which suppresses the hyperexcitability defects in mutants such as $S h$ and $H k$, as well as bang sensitivity (Ganetzky and Wu, 1982a,b; Jackson et al., 1985).

$b s s ;$ mlenapts double mutants were constructed by crosses between $g$ $b s s^{M W /} f$ or $b s s^{2} f$ and $m l e^{\text {nippsi }} c n$ flies with subsequent selection for F2 $f ; m l e^{\text {napts }} \mathrm{cn}$ or $g f ;$ mle napts $\mathrm{cn}$. The presence of bss was confirmed by back crosses to bss flies. bss; mlenants flies had a markedly lower viability and fertility than either $b s s$ or mlenturs alone (Reynolds, personal com-

Table 1. Genotypes of the mutants used, along with an indication of the nature of the defect and the molecular identity of the relevant gene product, if known

\begin{tabular}{|c|c|c|c|c|}
\hline Locus & Genotypes used & Affected gene product & Phenotype & Reference \\
\hline easily shocked & $e a s^{P C 80}, w e a s^{P C 80} f$ & Ethanolamine kinase & bang-sensitive & Pavlidis et al., 1994 \\
\hline bang senseless & $b s s^{M W 1} f, g b s s^{2} f$ & $?$ & bang-sensitive & Ganetzky and Wu, 1982a \\
\hline bang sensitive & bas, bas; ry $y^{506}$ & $?$ & bang-sensitive & Grigliatti et al. 1973 \\
\hline knockdown & y cho $c v k d n f$ & $?$ & bang-sensitive & Ganetzky and Wu, 1982a \\
\hline technical knockout & $t k o^{25 t}$ & Mito. ribosomal protein & bang-sensitive & Royden et al., 1987 \\
\hline slamdance & $s d a^{i s o 7.8}$ & $?$ & band-sensitive & Present results \\
\hline $\mathrm{Na}^{+}-\mathrm{K}^{+}$ATPase & $\mathrm{P}\left[r y^{+}\right] 2206 r y^{506}$ & $\mathrm{Na}^{+}-\mathrm{K}^{+}$ATPase & bang-sensitive & Schubiger et al., 1994 \\
\hline Shaker & $S h^{K S / 33}, S h^{r K 0120}$ & $\mathrm{~K}^{+}$channel & leg-shaker & Kamb et al., 1988 \\
\hline Hyperkinetic & $H k^{\prime}$ & $?$ & leg-shaker & Stern and Ganetzky, 1989 \\
\hline Frequenin & $\mathrm{T}(1 ; \mathrm{Y}) \mathrm{V} 7, y w f . B^{s} y^{+}$ & $\mathrm{Ca}^{2+}-$ binding protein & leg-shaker & Pongs et al., 1993 \\
\hline seizure & $s e i^{s s}$ & $?$ & ts paralytic & Jackson et al., 1985 \\
\hline paralyzed & $\operatorname{para}^{s s t}$ & $\mathrm{Na}^{+}$channel & ts paralytic & Loughney et al., 1989 \\
\hline shibire & $s h i^{i s t}$ & Dynamin & ts paralytic & van der Bliek and Meyerowitz, 1991 \\
\hline $\begin{array}{l}\text { male lethal-no } \\
\quad \text { action potential }\end{array}$ & $m l e^{\text {napss } 1} \mathrm{cn}$ & RNA helicase & ts paralytic & Kernan et al., 1991 \\
\hline
\end{tabular}


Table 2. Numbers of flies tested and trials performed, along with data on the effects of buzzes on DLM responses

\begin{tabular}{|c|c|c|c|c|c|c|c|c|}
\hline \multirow[b]{2}{*}{ Strain } & \multicolumn{2}{|l|}{$N$} & \multirow[b]{2}{*}{ Seizures } & \multicolumn{3}{|l|}{ Short buzz } & \multirow[b]{2}{*}{$\begin{array}{l}\text { Long buzz } \\
\text { failure } \\
\text { duration } \\
(\mathrm{sec}) \\
\end{array}$} & \multirow[b]{2}{*}{$\begin{array}{l}\text { Refract- } \\
\text { oriness }\end{array}$} \\
\hline & Flies & Trials & & $\begin{array}{l}\text { Failures/ } \\
\text { short buzzes }\end{array}$ & $\begin{array}{l}\text { Failure } \\
\text { duration } \\
(\mathrm{sec})\end{array}$ & $\begin{array}{l}\text { Failures/ } \\
\text { long } \\
\text { buzzes }\end{array}$ & & \\
\hline eas & 44 & 176 & ++++ & $150 / 237(70 \%)$ & $86 \pm 46$ & ND & & ++ \\
\hline bss & 14 & 65 & $+t+t$ & $58 / 68 \quad(85)$ & $112 \pm 70$ & ND & & +++ \\
\hline bas & 11 & 39 & +++ & $41 / 62 \quad(66)$ & $46 \pm 20$ & ND & & ++++ \\
\hline tko & 10 & 40 & +++ & $36 / 61 \quad(59)$ & $54 \pm 14$ & ND & & ++++ \\
\hline$k d n$ & 10 & 40 & $++t$ & $33 / 57 \quad(58)$ & $58 \pm 20$ & ND & & $+t+t$ \\
\hline$s d a$ & 5 & 19 & $+t+t$ & $19 / 29 \quad(66)$ & $88 \pm 37$ & ND & & ++ \\
\hline eas; mle $e^{\text {napts }}$ & 5 & 27 & +++ & $18 / 49$ & $27 \pm 16$ & ND & & ND \\
\hline bss; mlenamts & 6 & 26 & ++++ & $22 / 32 \quad(68)$ & $37 \pm 15$ & ND & & ND \\
\hline$m l e^{\text {napts }}$ & 6 & 25 & - & $19 / 46 \quad(41)$ & $42 \pm 33$ & $6 / 6(100 \%)$ & $25 \pm 9$ & - \\
\hline 2206 & 19 & 67 & + & $44 / 83 \quad(53)$ & $32 \pm 15$ & $7 / 16(43)$ & $22 \pm 16$ & - \\
\hline Frq & 7 & 20 & - & $7 / 44 \quad(16)$ & $15 \pm 13$ & $21 / 30(70)$ & $41 \pm 12$ & - \\
\hline$H k$ & 11 & 36 & $-1+++a$ & $9 / 104(9)$ & $15 \pm 11$ & $29 / 35(82)$ & $30 \pm 7$ & - \\
\hline$S h$ & 5 & 10 & - & $6 / 24 \quad(25)$ & $17 \pm 9$ & $7 / 8(88)$ & $22 \pm 12$ & - \\
\hline parat $^{t s}$ & 4 & 21 & - & $23 / 44 \quad$ (53) & $12 \pm 9$ & $13 / 16(81)$ & $18 \pm 10$ & ND \\
\hline$s e i^{\prime s}$ & 4 & 19 & - & $8 / 69 \quad(12)$ & $4 \pm 3$ & $15 / 23(65)$ & $21 \pm 9$ & ND \\
\hline$s h i^{t s}$ & 4 & 8 & - & $8 / 29 \quad(28)$ & $13 \pm 11$ & $4 / 4(100)$ & $16 \pm 17$ & ND \\
\hline wild type & 16 & 48 & - & $9 / 36 \quad(25)$ & $6 \pm 7$ & $59 / 78(74)$ & $28 \pm 13$ & - \\
\hline
\end{tabular}

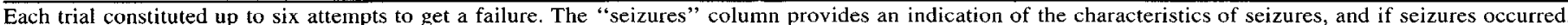

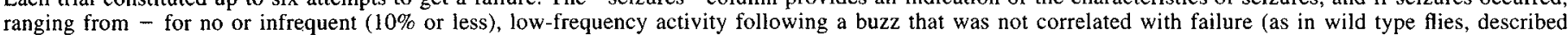

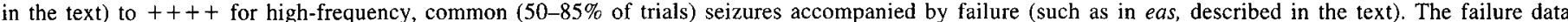

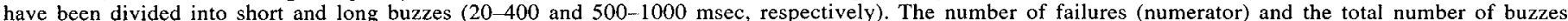

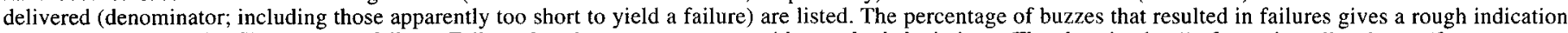

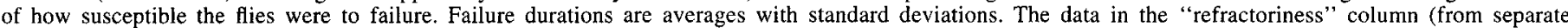

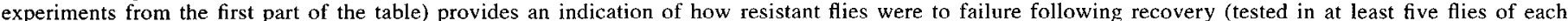

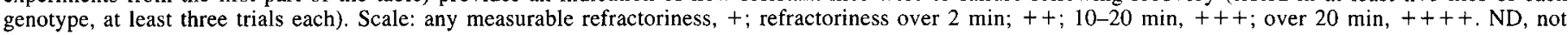
determined.

The $-1+++$ for $H k$ indicates that seizures were only observed in $H k$ flies following long buzzes.

munication). In a similar fashion, eas; mlenapts double mutants were constructed by crosses between eas $f$ and $m l e^{\text {napss }} c n$ flies with subsequent selection for $\mathrm{F} 2 f ; m l e^{n a p t s} \mathrm{cn}$ flies. Flies of the appropriate phenotype were recovered at a very low rate, were sterile, behaviorally sluggish and uncoordinated, and died within a week of eclosion. Because of the sterility of eas; mlenpis double mutants, we were unable to perform back-crosses to confirm the presence of eas in these flies at the behavioral level, though its presence at the physiological level was apparent (see Results). It is not yet clear if the effects of these double mutant combinations on viability are due to mlenaptsl or another locus on the mle ${ }^{\text {naptsl }} \mathrm{cn}$ chromosome.

Basic electrophysiology techniques. The GF pathway is illustrated in Figure 1. The basic method used to record GF-driven muscle potentials and to induce paralysis with electrical stimulation (a "buzz") was as described (Tanouye and Wyman, 1980; Pavlidis et al., 1994), with modifications as noted below and in the following sections. A fy is affixed to the recording stage in a position that does not interfere with wing or limb mobility. All stimulating and recording electrodes were uninsulated sharpened tungsten wires (in a small set of experiments testing the effects of buzzes on eas, similar results were obtained with $3 \mathrm{M} \mathrm{KCl}$ filled glass recording electrodes and stimulation with insulated tungsten wires under saline). Bipolar stimulating electrodes were inserted into the brain for the purposes of delivering GF stimuli and buzzes; a ground electrode was inserted in the abdomen. Two recording electrodes were used to measure potentials from the desired combination of ipsilateral DLMs (usually fibers 3 or 4 and 5 or 6 ), DVMs, and the TTM. Responses were recorded on a digital storage oscilloscope (Hitachi) and photographed.

Once stable muscle potential responses to suprathreshold test pulses (typically 10-30 V, $0.05 \mathrm{msec}$, delivered at $1 \mathrm{~Hz}$ ) delivered to the GF, an electrical "buzz" was delivered with a separate stimulator (Dagan S-900) to the brain via the same simulating electrodes, in the form of a short train of relatively high-voltage stimuli. Typically, we used 100 msec trains of $50 \mathrm{~V}, 200 \mathrm{IIz}$ stimuli, with a $0.5 \mathrm{msec}$ pulse duration. The duration of buzz, which could give rise to seizure and failure, was determined empirically for each bang-sensitive fly, and could be as brief as a single stimulus pulse in some eas flies (not shown). Buzz voltage had to be well above GF threshold to get failures in bang sensitives, suggesting that other neurons besides the GF must be stimulated to cause seizure and failure. If a buzz did not cause response failure, the buzz was increased in duration (often by manually triggering two or three 100 msec buzzes in a row, counted as a single buzz) and repeated, until five or six attempts had been made or until failure occurred. Each attempt, including unsuccessful ones, was included in the calculation of percent failures in Table 2 .

Following a buzz, GF stimulation at $1 \mathrm{~Hz}$ was resumed to test for the failure and then recovery of evoked muscle potentials. Recovery from failure is defined here by two successive GF test stimuli giving rise to normal (wild-type) latency responses ( $1.25 \mathrm{msec}$ for the DLMs; $0.8 \mathrm{msec}$ for the TTM; $\sim 2-3 \mathrm{msec}$ for the DVMs, Tanouye and Wyman, 1980 ) in both fibers being recorded from. Flies were rested at least 15 min between testing in this manner to allow recovery from refractoriness (see Results). Each set of buzzes separated by rest periods constituted a trial. Longer rests were used for bang sensitives with longer refractory periods ( $t k o, k d n$, and bas; bas required rests of 30-60 min because of refractoriness).

Although all the recordings reported in this article were from muscle fibers, we take the DLM potentials we recorded to reflect motor neuron action potentials on a one-to-one basis, as has been previously demonstrated (Koenig and Ikeda, 1983). Thus, seizure-like activity in the DLM reflects seizures in the DLMmns. This assumption is also supported by the fact that Drosophila muscles are not electrogenic except under extreme experimental conditions of prolonged depolarization (Salkoff and Wyman, 1980), and even then the spikes have a characteristic shape and time course (Salkoff and Wyman, 1980) not observed in the bang-sensitive seizures, during which potentials have the shape and time course of a synaptically evoked potential (Pavlidis, 1994). Thus, the seizures are unlikely to arise from intrinsic muscle membrane hypcrexcitability. Finally, muscle fibers 5 and 6 , which are innervated by the same motor neuron, maintain entirely synchronous activity even 
A

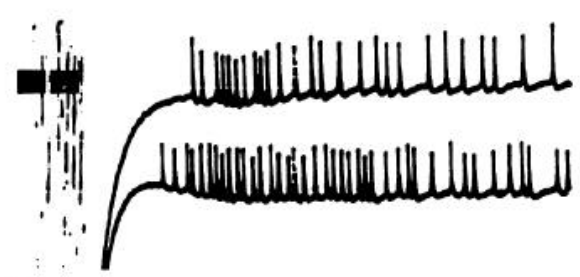

D

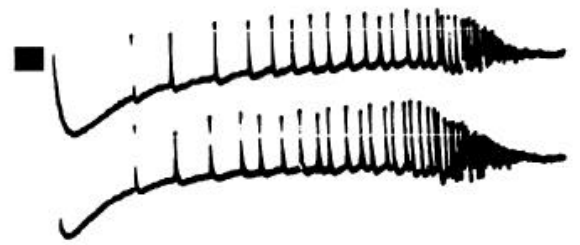

B

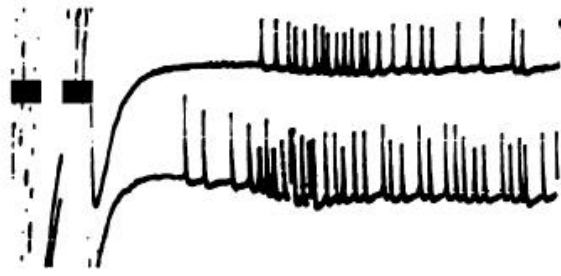

$\mathrm{E}$

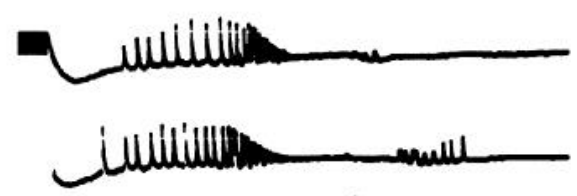

$\mathrm{C}$

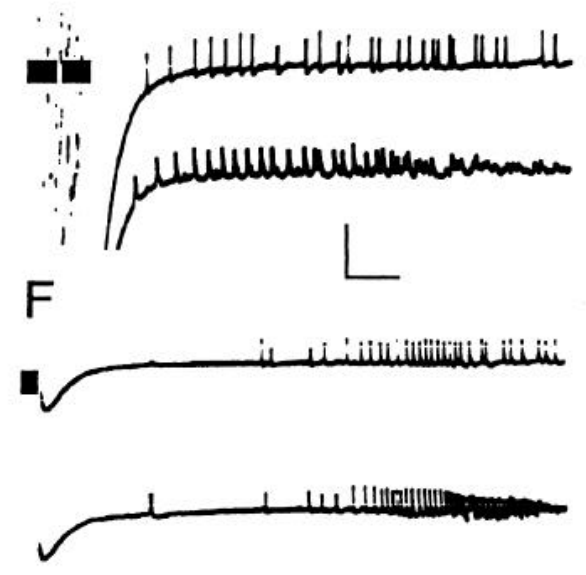

Figure 2. Examples of seizures from kdn and eas. The pair of traces in each panel are recordings from two ipsilateral DLM fibers. The short black bars indicate the timing and extent of buzzes, which generate a large stimulus artifact visible in the beginning of some of the traces (in some cases, multiple $100 \mathrm{msec}$ buzzes were used). $A-C, k d n . D$ and $E$ are typical of $k d n$ seizures, which did not generally ( $>75 \%$ of trials) decrease in potential amplitude or increase in frequency. An exception is shown in $C$ (bottom trace). $D-F$, eas. $A$ and $B$ are typical of eas seizures. Note the increase in frequency and decrease in potential amplitude as the seizures progress, seen in at least $90 \%$ of eas seizures. The example in $F$ is unusual for eas in that the seizure in one DLM fiber (top trace) appears to die out without increasing in frequency greatly or decreasing in potential amplitude. $F$ also demonstrates a relatively long pause between the buzz and the seizure. Calibration: $20-50 \mathrm{mV}, 200 \mathrm{msec}$.

during intense seizures (not shown), consistent with seizures being neuronally derived. Thus, we use the term "seizure" to denote uncontrolled, high-frequency neuronal activity, though we assay it indirectly with muscle potential recordings.

Motor neuron stimulation. In experiments requiring direct stimulation of motor neurons, a second set of stimulating electrodes driven by a third stimulator (Grass S44) was employed. These electrodes had rightangle bent tips and were positioned in the anterior preepisternum (near the base of the first coxa) with the aid of a mirror placed under the fly. As the stimulus voltage is increased, a GF-initiated TTM or DLM potential was first elicited at $\sim 1 \mathrm{~V}, 0.05 \mathrm{msec}$ duration, with a shift to a shorter latency $(\sim 0.6 \mathrm{msec}$ for the TTM, $\sim 0.8 \mathrm{msec}$ for the DLM) at higher voltages (typically $\sim 3 \mathrm{~V}$ ); this short latency response is due to direct stimulation of the motor neurons (Salkoff and Kelly, 1976).

Identification of recording sites. When identified recording sites in DLM fibers 5 and 6 were needed, electrode placement was guided by cuticular markers (Levine and Hughes, 1973) and confirmed by the following method, modified from Engel and Wu (1992). After removing the electrodes, a drop of a $2 \%$ solution of methylene blue in phosphate buffered saline (PBS) containing $0.1 \%$ Tween 20 (to improve wetting) was applied to the dorsal thorax and the fly placed in a humidified chamber. After 10-30 min, the fly was transferred to a solution of $4 \%$ formaldehyde, $4 \%$ DMSO in PBS for 4-6 hr. This procedure leaves a small blue spot on the cuticle at the electrode insertion sites and allows identification of the underlying fiber by dissection.

\section{Results}

\section{Seizure and failure in all bang-sensitive mutants}

Electrical buzzes (50-400 msec) applied to the brain of bangsensitive mutants reveals a complex physiological defect. In most of the experiments to be described this was monitored by recordings of DLM potentials. The first aspect of the physiological phenotype is abnormal spontaneous activity (a "seizure") following the delivery of the buzz. Seizures were observed in every bang-sensitive fly, after $60-85 \%$ of buzzes. The seizure is likely to correspond to the preparalysis hyperactivity phase observed in these mutants (Royden, et al., 1987; Pavlidis et al., 1994).

DLM seizures lasted $\sim 0.5-3 \mathrm{sec}$. Examples of traces demonstrating the variability of seizure appearances are given in Figure 2. From this figure, it can be seen that seizures fell into two loose classes. The first class is exemplified by the seizures seen in at least $75 \%$ of trials with $k d n$ flies (Fig. $2 A-C$ ). These seizures typically began with trains of DLM potentials at $\sim 10$ $30 \mathrm{~Hz}$, which lacked any clear pattern. As the seizures progressed, the frequency of activity changed little until dying out. The second class is exemplified by at least $90 \%$ of trials with eas flies (Fig. $2 D-F$ ). These seizures began in a manner similar to those in the first class, but soon show a marked increase in frequency with a concomitant decrease in potential amplitude. The frequency increase and amplitude decrease continued until the potentials were undetectable. Examination of seizures at faster sweep speeds than shown here revealed that the activity near the end of this type of seizure could reach $150 \mathrm{~Hz}$. Examples of typical seizures from the other bang-sensitive mutants (bss, bas, $t k o$, and $s d a$ ) are shown in Figure 3.

The next aspect of the phenotype is the sudden failure of GF stimulation to evoke DLM potentials following the buzz. Such failure in the nervous system is a likely underlying cause of behavioral paralysis in these mutants. Failure occurred in every bang-sensitive fly tested, after $60-85 \%$ of buzzes (Table 2 ). There was considerable variation in the duration of failures in the different bang sensitives, ranging from an average of about $45 \mathrm{sec}$ in bas to $112 \mathrm{sec}$ in bss (Table 2). In all the bang sensitives, there was a close correlation between the occurrence of seizures and failure: if there was a seizure, there was a failure $>95 \%$ of the time. Failures occurred without seizures in about $10 \%$ of cases.

\section{Additional common aspects of the bang-sensitive physiological phenotype}

In agreement with their behavioral similarity, the bang sensitives had many similarities in their physiological defects in addition to seizure and failure. First, the DLM failure period consisted of two distinct phases in all the bang sensitives: a silent period, with no evoked or spontaneous activity, followed by a period of spontaneous activity. The silent period had a very consistent duration of $\sim 35-40 \mathrm{sec}(34 \pm 6 \mathrm{sec}$ in bss, $35 \pm 6 \mathrm{sec}$ in eas, $37 \pm 6 \mathrm{sec}$ in bas, $38 \pm 7 \mathrm{sec}$ in $s d a, 41 \pm 8 \mathrm{sec}$ in $k d n$, and 

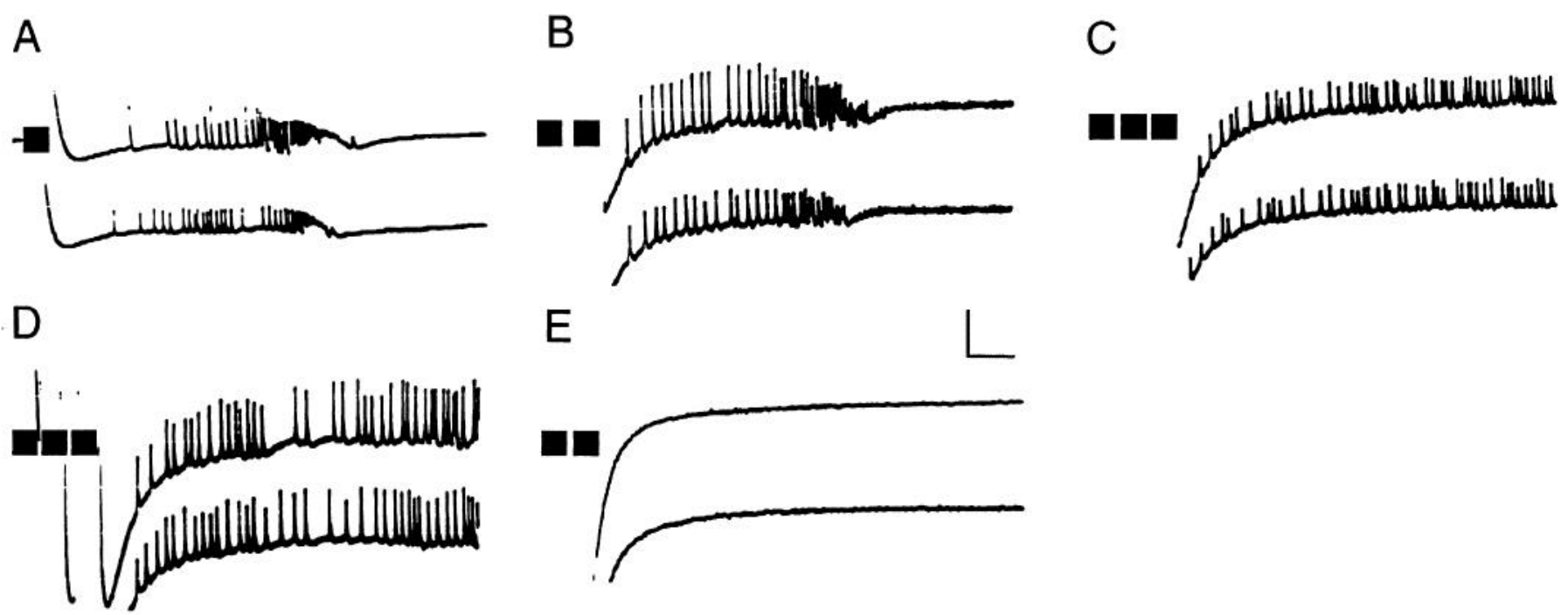

Figure 3. Typical seizures from bss, sda, bas, and tko. A, bss; B, sda; $C, b a s ; D, t k o, E$, control (wild-type). For each bang-sensitive mutant, the example shown is representative of the seizures observed in at least $80 \%$ of trials. Black bars indicate buzzes as in Figure 2 . Calibration: $20 \mathrm{mV}$, $200 \mathrm{msec}$.

$42 \pm 3 \sec t k o ; n=12-27$ trials on four to six flies for each mutation). This silent period probably corresponds to the paralysis phase of the behavior, which lasts 30-40 sec (Pavlidis, 1994; E. R. Reynolds and P. Pavlidis, unpublished observations). The subsequent spontaneous DLM activity varied greatly in intensity from fly to fly and trial to trial, but often resembled the seizure (not shown). During this period, evoked responses were still absent. This phase apparently reflects the behavioral hyperactivity observed during recovery from behavioral paralysis (Royden et al., 1987; Pavlidis et al., 1994).

Second, abnormal evoked responses were observed during recovery. In wild-type flies, and in bang sensitives prior to a buzz, GF stimulation always gives rise to synchronized responses in all the DLM fibers (Fig. 4A,B; Tanouye and Wyman, 1980). However, in all bang sensitives tested, evoked potentials from individual DLM fibers appeared independently and irregularly during recovery, in $>95 \%$ of trials. Examples of independent recovery are shown for each bang sensitive in Figure 4 . Additionally, in at least $50 \%$ of all trials in all the mutants, the DLM responses early in recovery had abnormal, long, variable latencies. The latencies were commonly different for the two DLMs being studied, as in Figure $4 E$; abnormal latency is also apparent in Figure $4 F$. In $\sim 10 \%$ of trials, abnormal-looking potential amplitudes and shapes were also observed (not shown). As recovery proceeded, these abnormalities became progressively less severe (e.g., latencies became gradually shorter and responses) until reaching a normal state (as in Fig. $4 A, B$ ).

A third common aspect of the defect was a refractory period following recovery, during which a buzz was less effective at producing seizures and failure (Table 2). This is likely to reflect the behavioral refractoriness that has been previously noted in all bang sensitives (Ganetzky and Wu, 1982a). For example, we found it necessary to rest bas flies at least $30 \mathrm{~min}$ between trials before buzzes could induce seizure and failure again $(n=5$ flies, at least three trials each), in agreement with observations that bas flies are refractory to vortexing for up to an hour (Grigliatti et al., 1973). In eas flies buzzing immediately following recovery usually did not cause seizure or failure, but short rests (2-10 $\mathrm{min}$ ) restored the ability of a buzz to cause seizure and failure ( $n=5$ flies, at least three trials each); again, this agrees well with behavioral observations (Pavlidis et al., 1994).

The conclusions from these experiments are that all six bangsensitive mutants have qualitatively similar phenotypes at the level of the DLM responses to a buzz, and furthermore, that this syndrome seems to reflect all the different stages of the bangsensitive behavioral phenotype, including preparalysis hyperactivity, paralysis, postparalysis hyperactivity, recovery, and the refractory period (Pavlidis et al., 1994). On this basis, we suggest that the response of the DLM to a buzz is probably representative of what occurs in many other parts of the bang-sensitive nervous system following a bang, and can thus be used as a starting point in analyzing the physiological basis of bang sensitivity.

\section{Failure of synaptic transmission in the GF-DLM pathway}

We next asked whether the failure in the GF pathway is due to failure of the GF itself or to other elements in the pathway. The independent recovery of GF-evoked DLM potentials following a buzz (Fig. 4) suggests that failure has occurred downstream of the functional branch point in the pathway. This indicates that the limiting site of recovery in the GF pathway is either the peripherally synapsing interneuron (PSI)-DLM motor neuron (DLMmn) synapse, the DLMmn action potential, or the DLM $\mathrm{nmj}$, and not at the level of the GF or PSI action potentials, which would result in all-or-none recovery in all six DLM fibers (Fig. 1).

The spontaneous activity during recovery suggests that DLMmns can still fire action potentials while GF-evoked responses are still absent. To confirm this, we directly stimulated the motor neurons of eas flies by placing a second set of stimulating electrodes in the thorax ( $n=15$ flies, 46 trials). As expected, GF stimulation failed to yield DLM responses after a buzz (Fig. 5A) until recovery occurred with the time course expected for eas $(60-100 \mathrm{sec})$. During this period, stimulation of the motor neurons still evoked DLM responses (Fig. 5A), although in $30 \%$ of trials, the responses did fail briefly, or the DLM potentials changed size. Such mn-evoked response changes lasted an average of $5 \mathrm{sec}$, and persisted for no more 

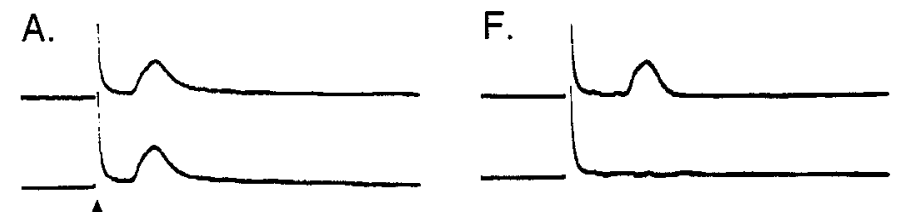

B.

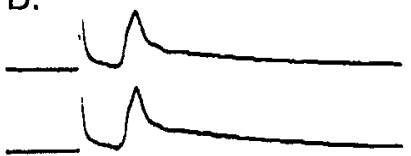

G.

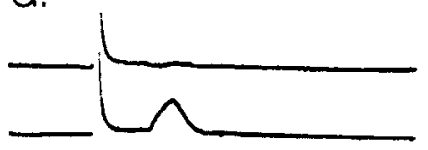

C.
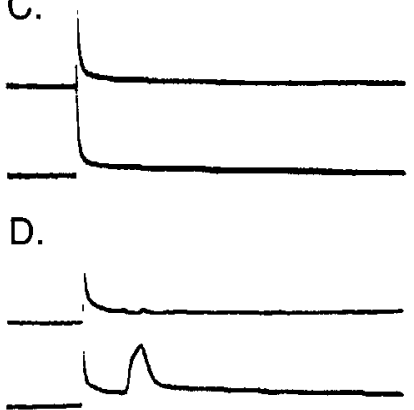

E.

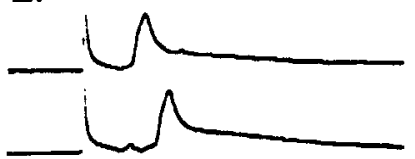

$\mathrm{H}$.

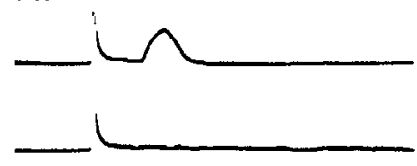

I.

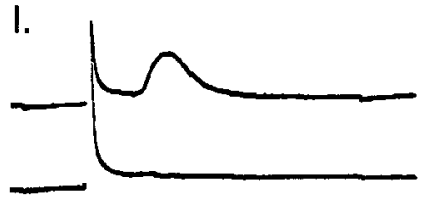

J.

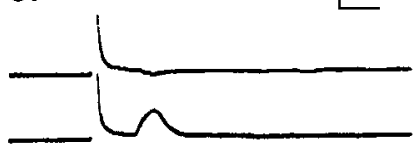

Figure 4. Abnormal DLM responses following buzzes in bang sensitives. A, Wild-type DLM recordings showing the normal, synchronous response to GF stimulation (the stimulus artifact is indicated by an arrowhead), with a invariable latency $(1.2 \mathrm{msec}$; Tanouye and Wyman, 1980). $B$, Normal responses in eas prior to a buzz. These responses are indistinguishable from those in wild-type flies, as shown in $A$ (other bang-sensitive mutants also have wild-type-like responses before a buzz, not shown). $C$, Failure of GF stimulus-evoked DLM responses in eas following a buzz, typical of what was observed in all bang-sensitive flies. $D$, Independent recovery of individual DLM fibers in eas, indicating failure at a branch point in the GF pathway. $E$, Abnormally long, independently varying DLM response latencies during recovery in an eas fly. $F-J$, Examples of independent recovery of individual DLM fibers in $t k o, k d n, s d a, b s s$, and bas, respectively. Calibration: $50 \mathrm{mV}$, 2 msec.

than $10 \mathrm{sec}$. Similar results were obtained in bss flies $(n=2$ flies, six trials). This shows that for most of the failure period, the DLMmns, the DLM neuromuscular junctions, and the DI Ms are functional. It confirms that the site in the pathway limiting recovery lies upstream of the DLMmn action potential.

In another set of experiments, we recorded simultaneously from eas DLM fibers 5 and 6, which are innervated by the same motor neuron (Fig. 1). In this case, recovery was never independent ( $n=10$ trials in four eas flies; compared to 13 independent recoveries in 14 trials in four eas flies when recording from other muscle pairs). This is further evidence that the site of failure is not individual DLM nmjs. More importantly, it shows that independent recovery is not an artifact caused by an effect on the DLMmns (i.e., irregular transmission), but is, in fact, due to a defect upstream in the pathway. The only points upstream of the DLMmn action potentials that could result in independent recovery of DLMs are the PSI-DLMmn synapses (Fig. 1). The changes in response latency commonly observed during recovery (Fig. 4) are also consistent with PSI-DLMmn synaptic failure.
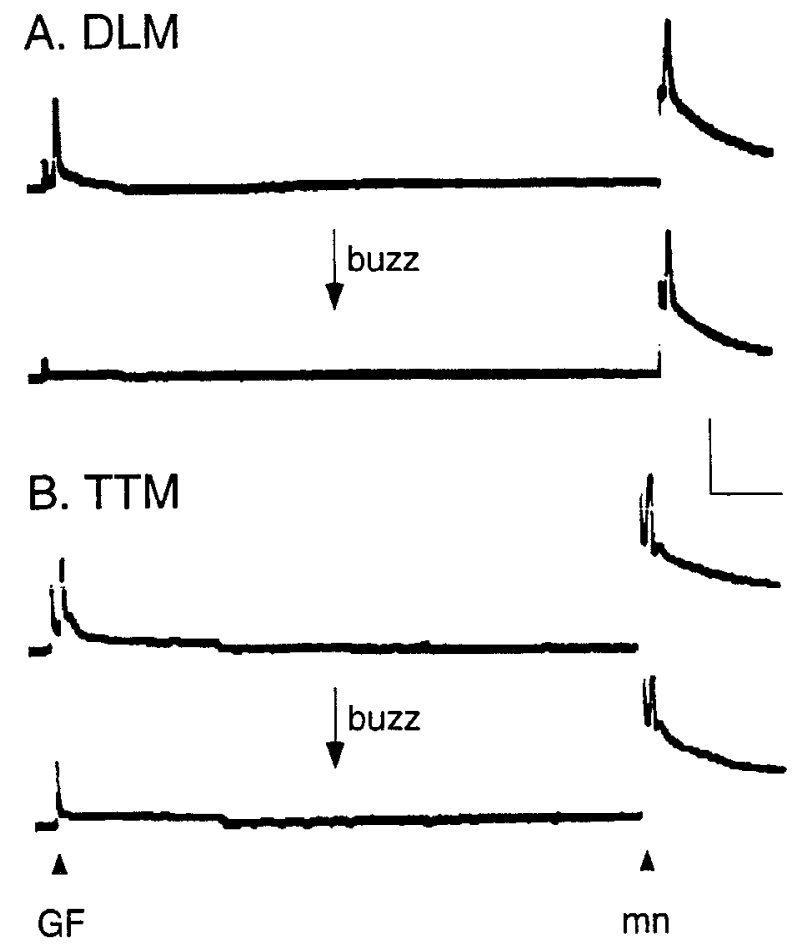

Figure 5. Motor neuron function during GF-evoked response failure. For each trace, a stimulus (indicated by arrowheads at bottom) was applied first to the brain to evoke a response initiated at the GF, then, after a pause of $\sim 100 \mathrm{msec}$, to the thoracic ganglion to evoke a response initiated at the motor neuron (the mn-evoked response rides on a large stimulus artifact). Typical responses before (top traces of each pair) and after a buzz (bottom traces of each pair) are shown. Identical results were obtained if the stimulus order was reversed (thoracic stimulus first; not shown). Between each such pair of test pulses there was a $1 \mathrm{sec}$ rest. $A$, Recording from a DLM. After a buzz, GF stimulation fails to crokc a DLM response, but motor ncuron stimulation docs. $B$, Recording from a TTM (a different fly than in $A$ ). As for the DLM motor neuron, stimulation still evokes a DLM response after a buzz. Calibration: $A, 50 \mathrm{mV}, 20 \mathrm{msec} ; B, 20 \mathrm{mV}, 20 \mathrm{msec}$.

\section{Failure in other $G F$-activated muscles}

If the events observed in the DLM reflect a widespread phenomenon in the fly, one would expect other muscles in the fly to undergo seizures and failure following a buzz. As a first examination of this hypothesis, we tested the effects of buzzes on the other muscles which respond to GF stimulation, the dorsal ventral indirect flight muscle (DVM), and the tergotrochanteral jump muscle (TTM) (Fig. 1). These studies were carried out primarily on eas flies, with some experiments on bss. Delivery of a buzz causes the DVM fibers to undergo a seizure that appears qualitatively similar to that in the DLMs (Fig. 6, top). In contrast, seizures were never observed in the TTM (Fig. 6, bottom). Following the buzz, the DVMs and the TTM fail to respond to GF stimulation. Recovery of the DVMs followed a very similar time course as the DLMs (eas: $92 \pm 27 \mathrm{sec}, n=$ 5 flies, 10 trials; $b s s: 111 \pm 71 \mathrm{sec}, n=3$ flies, 8 trials). The TTMs recovered somewhat more quickly, in an average of 73 $\pm 33 \mathrm{sec}$ in eas ( $n=15$ flies, 46 trials), and in $66 \pm 21 \mathrm{sec}$ in bss ( $n=5$ flies, 15 trials). Also, in $58 \%$ of the trials with eas, TTM responses that were normal in appearance and usually normal in latency bricfly reappeared much carlicr than DLM or DVM recovery. In bss, early recovery occurred in $40 \%$ of the trials. Early recovery was always coincident with the beginning of spontaneous activity ( $30-40 \mathrm{sec}$ after the buzz). These early 


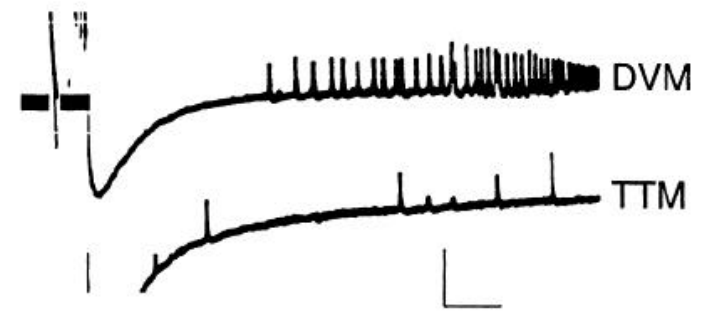

Figure 6. Responses of DVM and TTM to buzzes in eas. The DVM (top trace) undergoes a seizure like that observed in the DLMs, while the TTM (bottom trace) does not. This example is atypical since there was some TTM activity, but was selected to show that TTM potentials could be recorded. Similar results were obtained with bss flies. Buzzes are indicated as in Figure 2. Calibration: DVM, $20 \mathrm{mV}, 200 \mathrm{msec}$; TTM, $10 \mathrm{mV}, 200 \mathrm{msec}$.

responses were unstable (in all but five eas trials), decreasing in amplitude and disappearing, recovering again with a normal time course (not shown). We also tested TTM motor neuron (TTMmn) function following buzzes in eas. As for the DLMmn, TTMmn stimulation could still yield a normal evoked TTM response (Fig. $5 B$ ). Failures of the TTMmn- derived response occurred more frequently than for the DLMmn (56\% of TTMmn trials vs $30 \%$ of DLMmn trials). Failures of the TTMmn-derived response were also much longer lasting (TTMmn average failure $18 \mathrm{sec}, n=6$ flies, 19 trials; DLMmn average failure $5 \mathrm{sec}, n$ $=15$ flies, 46 trials). These results indicate that the effects of buzzes on the GF-TTM pathway may be more complex than those on the GF-DLM (see Discussion).

\section{Relationship between seizure and failure}

A simple explanation for the cause of failure is that the intense activity of the seizure could result in the inactivation of labile sites in neurons, such as the PSI-DLMmn synapses. However, as described above, DLM failures sometimes occurred without the observation of a seizure (that seizures do not occur in the TTM also demonstrates that seizures are not an obligate feature of the phenotype). This shows that PSI synaptic failure is independent of seizures in the DLMmns, and raises the question of whether PSI seizures occur at all.

We further explored the relationship between seizure and failure by testing the effects of a suppressor of bang sensitivity, $m l e^{\text {napts }}$, on bang-sensitive physiology. Since mle $e^{\text {napts }}$ decreases neuronal excitability (Wu and Ganetzky, 1980), we hypothesized that mlents might suppress seizures in double mutant combination with bang-sensitive mutations. However, buzzes could still induce DLM seizures in $b s s ; m l e^{\text {napts }}$, while failures were greatly reduced in duration (Table 2; similar results were obtained using either $b s s^{s w \prime}$ or $b s s^{2}$ ). The seizures were qualitatively similar to those seen in bss alone (Fig. 7A). Even more dramatic than the effects on bss, in eas; mle napts seizures were not consistently accompanied by failures ( 39 seizures but only 18 failures in five flies). Again, seizures were not obviously reduced in severity (Fig. 7B). Failures in these flies, when they occurred, were also reduced in duration (Table 2). Thus, seizures in the DLMs are at least partially separable from PSI synaptic failure in these double mutants.

Alone, mle $e^{\text {napts }}$ DLMs did not have seizures following buzzes, although there were sometimes significant failures (Table 2). These failures were distinct from bang-sensitive failures in that there was no refractory period $(n=14$ tests of refractoriness on
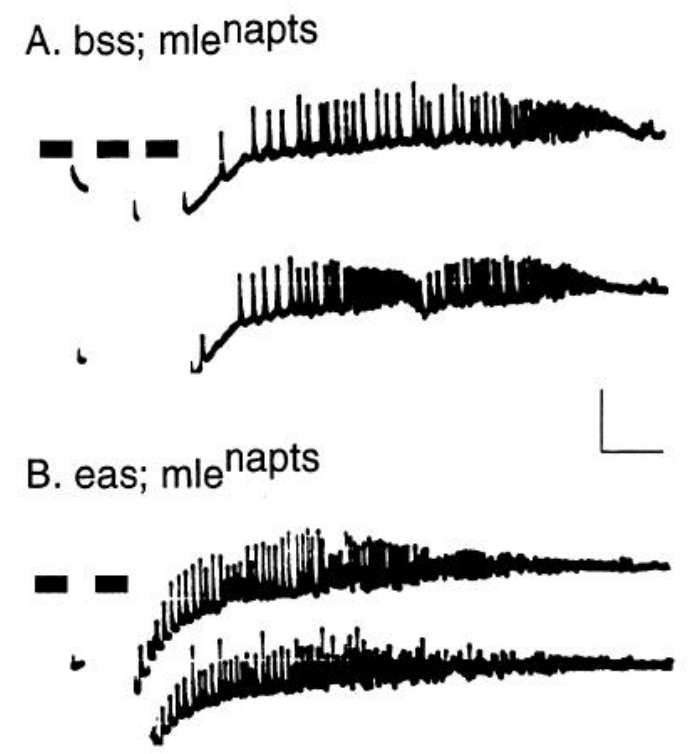

Figure 7. Seizures in bss; mle napts and eas; mlentapts double mutants. A, bss; mle $e^{\text {napts }} ; B$, eas; mle mapts $^{\text {. The }}$ The seizures are indistinguishable from those seen in bss and eas. Calibration: $20 \mathrm{mV}, 200 \mathrm{msec}$.

two flies), spontaneous activity during recovery, or independent recovery.

\section{Effects of buzzes on other excitability mutants}

In wild-type flies (Pavlidis et al., 1994), short buzzes $(<500$ msec) have no effect on the DLM responses other than an occasional slight, temporary threshold increase. To examine the possibility that seizures and failures might not be specific to bang sensitives, we tested the responses of a number of other excitability mutants to short buzzes. The results are summarized in Table 2. We tested the three mutants ( $\mathrm{Sh}, \mathrm{Hk}, \mathrm{Frq}$ ), which have larval nmj defects similar to bss. We also tested several temperature-sensitive paralytic mutants, $s e i^{t s}, p a r a^{t s}$, and $s h i^{t s}$. There was no significant effect of short buzzes on any of these mutants (Table 2).

We tested the 2206 strain (the $\mathrm{Na}^{+}-\mathrm{K}^{+}$ATPase mutant), which has a mild mechanical shock-sensitive behavioral phenotype (Schubiger et al., 1994). The 2206 behavioral phenotype is significantly different from the bang sensitives; for example, they are paralyzed for only a few seconds and lack a refractory period (Pavlidis, 1994; Schubiger et al., 1994). We found that in many 2206 flies, short buzzes could cause failure lasting $\sim 30 \mathrm{sec}$ (Table 2). Such failures were sometimes accompanied by activity following the buzz ( $20 \%$ of trials) (Fig. $8 A$ ); however, spontaneous activity could also occur following single test stimuli and without failure (not shown).

We also examined the effects of longer buzzes on these excitability mutants, since in wild-type flies, long buzzes (500$1000 \mathrm{msec}$ ) can cause failures of about $30 \mathrm{sec}$ (Pavlidis et al., 1994; Table 2). Failures in wild-type flies were different from those in bang sensitives. First, there was spontaneous activity following only $\sim 10 \%$ of long buzzes, ranging from $1-10$ potentials to the initiation of flight-like activity $(\sim 10 \mathrm{~Hz})$, compared to the routine observation of seizures in the bang sensitives (after at least $60 \%$ of all buzzes). Such activity was not well correlated with failure (failure in only $\sim 50 \%$ of trials in which there was spontaneous activity; compared to a correlation of $>95 \%$ in the bang sensitives). There was also no refractory 
A. 2206

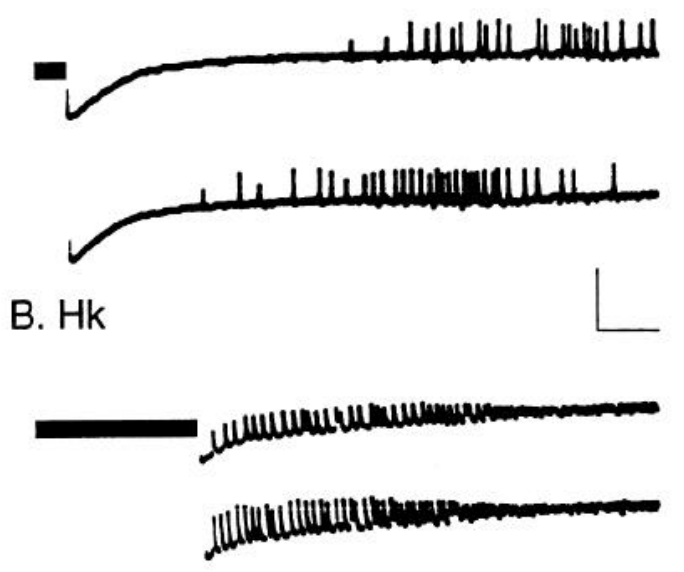

Figure 8. DLM hyperactivity following buzzes in non-bang-sensitive strains. A, DLM hyperactivity in a 2206 fly following a $100 \mathrm{msec}$ buzz. In 2206 flies, such activity was only seen after about $20 \%$ of buzzes, which resulted in failure. $B$, A $H k$ DLM seizure following a $500 \mathrm{msec}$ buzz. In this example, the potential size declined as the seizure progressed. Calibration: $50 \mathrm{mV}, 200 \mathrm{msec}$.

period for failures in wild-type flies, as a second buzz delivered immediately after recovery always causes a second failure $(n=$ 5 flies, at least three trials each).

With two exceptions, all the additional excitability mutants had responses to long buzzes indistinguishable from wild-type flies (Table 2). Frq mutant DLM responses failed for slightly longer periods after long buzzes as compared to wild-type flies ( $p=0.05$; Student's $t$ test), but did not have seizures. Interestingly, $H k$ flies had a much higher tendency for seizure-like activity following long buzzes than wild-type ( $\sim 70 \%$ of trials). Additionally, in several of the $H k$ flies examined, the DLM potentials decreased in size during the seizure, similar to what was observed in some bang sensitives (Fig. $8 B$ ). Failure durations following long buzzes in $H k$ were similar to wild-type flies (Table 2).

Because they displayed some aspects of the bang-sensitive phenotype (behaviorally or physiologically), we tested $\mathrm{Hk}, \mathrm{Frq}$, and 2206 flies for refractoriness following DLM response recovery. In each case, no significant refractoriness was detectable. After recovery from a first buzz, a second $500 \mathrm{msec}$ buzz still induced DLM failures in $H k, F r q$, and 2206 flies (tested in at least four flies each, three trials per fly). In addition, there was no measurable refractory period for failures following short buzzes in 2206 flies ( $n=8$ flies, at least three trials each).

\section{Discussion}

Bang sensitives: a distinct class of excitability mutants Historically, Drosophila excitability mutants have been classified as either hyperexcitable (e.g., Sh) or hypoexcitable (e.g., para) (Hall, 1985). The bang sensitives have been generally considered hyperexcitable. This is based on the observation of abnormally rapidly developing giant potentials at bss larval nmjs (Jan and Jan, 1978), the suppression of bang sensitivity by mle mapts $^{\text {. }}$ (which also suppresses hyperexcitability mutants such as $\mathrm{Sh}, \mathrm{Ga}-$ netzky and Wu, 1982a,b), and the behavioral hyperexcitability during recovery (Royden et al., 1987). The seizures we have observed are also indicative of hyperexcitability. Despite their apparent hyperexcitability, some aspects of the phenotype are more suggestive of hypoexcitability: paralysis [including temperature-sensitive paralysis (Ganetzky and Wu, 1982a; Royden, 1988), also found in hypoexcitable mutants such as mlenapts and para $\left.^{t s}\right]$, reduced mechanoreceptor responses to bristle deflection (Engel and $\mathrm{Wu}, 1994)$, and a 20 -fold decrease in the rate of spontaneous transmitter release observed in bss larvae (Jan and Jan, 1978). Although the work presented here tends to align the bang sensitives with the hyperexcitable mutants, the apparent activity dependence of the defect sets them apart. The cause of this activity dependence and the cellular and molecular events leading to seizures, failure, and refractoriness remain unclear.

\section{Relationship between bang sensitives and hyperexcitable mutants}

We found that some, but not all, aspects of the bang-sensitive physiological phenotype can be found in certain hyperexcitability mutants. Following long buzzes, $H k$ flies could have DLM seizures and Frq flies failed for slightly longer periods than wildtype flies. 2206 flies were more sensitive than wild-type flies to short buzzes. Although consistent with hyperexcitability in bang sensitives, the differences between these mutants and the bang sensitives makes it unclear whether these similarities to bang sensitives are merely superficial or if they represent similarities at the cellular or molecular level. Thus, it is difficult to determine on this basis if the molecular defects in $\mathrm{Hk}, 2206$, or Frq are relevant to those in the bang sensitives.

The 2206 strain warrants further discussion because of its mechanical shock sensitivity, which has been compared to a bangsensitive phenotype (Schubiger et al., 1994). This P-elementmediated mutation results in a two-thirds decrease in $\mathrm{Na}^{+}-\mathrm{K}^{+}$ ATPase $\alpha$-subunit protein levels and causes marked behavioral hyperexcitability (Pavlidis, 1994; Schubiger et al., 1994). Given their weak phenotype and lack of a refractory period, it is unclear whether they belong within the bang-sensitive class of mutants. Since we found some physiological similarities between 2206 and the bang sensitives, we consider this an open question. However, because of the weak 2206 phenotype, and that eas and tko encode other proteins, we consider it unlikely that the other bang sensitives affect the $\mathrm{Na}^{+}-\mathrm{K}^{+}$ATPase itself. It is possible, nevertheless, that other bang sensitives affect a process that is also sensitive to $\mathrm{Na}^{+}-\mathrm{K}^{+}$ATPase activity.

\section{Synaptic transmission defects in bang sensitives}

The independent recovery of the DLMs (Fig. 4) and the ability of motor neuron stimulation to elicit DLM responses following a buzz (Fig. 5) show that DLM failure occurs at the level of individual PSI-DLMmn synapses. Similar logic has been applied in determining the cause of independent DLM responses in two other (non-bang-sensitive) mutants, $C h a^{t s}$ and $g f A$ (Gorczyca and Hall, 1984; Thomas and Wyman, 1984). It is unlikely that a failure of action potentials to invade individual PSI synapses could explain our findings, since it has been shown in anatomical studies that the PSI-DLMmn synapses are highly localized in a nonbranching PSI process in the posterior dorsal mesothoracic nerve (PDMN) (legend to Fig. 1; King and Wyman, 1980).

The behavior of the TTM is more difficult to explain in terms of synaptic failure. That TTMmn stimulation could still evoke responses after GF-evoked responses have failed appears to indicate the GF as the primary site of failure, or less likely, the GF-TTMmn electrical synapse. It is conceivable that the GF does fail but that this is only limiting for recovery of the TTM, since DLM recovery was not limited by GF responsiveness. 
However, this does not explain the "early recovery" of TTM responses we saw in many experiments. Without more data, we consider it possible that the current model of the GF-TTM pathway is incomplete. There are already suggestions that there may be multiple TTM motor neurons (King and Wyman, 1980) and that there are multiple GF-TTM pathways (Krishnan et al., 1993). Resolution of these issues will be important to our understanding of TTM physiology in bang sensitives.

Despite these problems, currently the best explanation for our data is that in bang-sensitive adults synapses fail following a bang or buzz. At this time we cannot determine if the site of failure is presynaptic or postsynaptic. The defects in bss larvae, while not including synaptic failure, are indicative of a presynaptic component to the phenotype (Jan and Jan, 1978; Ganetzky and $\mathrm{Wu}, 1982 \mathrm{a}$ ). However, the relationships among adult motor neuron seizures, altered larval motor neuron excitability, and failure of the PSI-DLMmn synapses remains unclear.

\section{Seizures and the causes of failure}

Contrary to our initial expectation that seizures cause failure in bang sensitives, seizures were not an obligate feature of the defect, as evidenced by the occasional GF-DLM response failure without seizure, as well as by the lack of seizures in the TTM. This suggests that PSI failure can occur without a PSI scizure, since one might expect some of such activity to be transmitted to the DLM even in the absence of DLMmn seizures. The finding that mlents suppresses bang-sensitive failures, but not seizures, is another indication that seizures are not a prerequisite for failures. Further support for this idea comes from experiments showing that eas flies, which are also temperature sensitive, do not undergo behavioral hyperactivity prior to cold-induced paralysis (Pavlidis, 1994). These findings suggest that seizures and failures are independent manifestations of the defect. In any case, seizures that can be triggered by exogenous stimulation of the nervous system are a novel phenomenon in Drosophila. More work will be required to determine if there is any relationship between bang-sensitive seizures and seizures in mammals. That both a human epilepsy and a bang-sensitive mutation have been traced to mitochondrial translational machinery defects is additionally suggestive that there might be some connection between seizures in flies and in mammals (Royden et al., 1987; Shoffner et al., 1990).

\section{Conclusion}

The present work shows that all bang-sensitive mutants have similar, though not necessarily identical, adult physiological defects that can account for the behavioral phenotype. Despite their similarities, molecular studies have thus far indicated diversity in the gene products affected in these mutants (Royden et al., 1987; Pavlidis et al., 1994). We propose that the bang sensitives all affect a common complex physiological process, capable of being affected by a range of genetic defects but with a similar end effect. The molecular diversity of the genes affected might explain some of the differences in phenotypes among the bang sensitives (e.g., differences in refractory period and DLM failure durations). For example, some of the differences among the bang sensitives might exist because some mutants have more or less severe defects in the common process. Alternatively, there may be additional processes affected in some mutants but not in others. Answers to these questions must wait until the bang-sensitive physiological and molecular defects are bettei understood.

\section{References}

Benzer S (1971) From the gene to behavior. JAMA 218:1015-1022. Engel JE, Wu C-F (1992) Interactions of membrane excitability mutations affecting potassium and sodium currents in the flight and giant fiber escape systems of Drosophila. J Comp Physiol [A] 171:93-104.

Engel JE, Wu C-F (1994) Altered mechanoreceptor response in Drosophila bang-sensitive mutants. J Comp Physiol [A] 175:267-278.

Ganetzky B, Wu C-F (1982a) Indirect suppression involving behavioral mutants with altered nerve excitability in Drosophila melanogaster. Genetics 100:597-614.

Ganetzky B, Wu C-F (1982b) Drosophila mutants with opposing effects on nerve excitability: genetic and spatial interactions in repetitive firing. J Neurophysiol 47:501-514.

Gorczyca M, Hall JC (1984) Identification of a cholinergic synapse in the giant fiber pathway of Drosophila using conditional mutations of acetylcholine synthesis. J Neurogenet 1:289-313.

Grigliatti TA, Hall L, Rosenbluth R, Suzuki DT (1973) Temperaturesensitive mutations in Drosophila melanogaster. XIV. A selection of immobile adults. Mol Gen Genet 120:107-114.

Hall JC (1985) Genetic analysis of behavior in insects. Comp Insect Phys Biochem Pharmacol 9:287-373.

Ikeda K, Koenig JH, Tsuruhara T (1980) Organization of identified axons innervating the dorsal longitudinal flight muscle of Drosophila melanogaster. J Neurocytol 9:799-823.

Jackson FR, Gitschier J, Strichartz GR, Hall LM (1985) Genetic modifications of voltage-sensitive sodium channels in Drosophila: gene dosage studies of the seizure locus. J Neurosci 5:1144-1151.

Jan YN, Jan LY (1978) Genetic dissection of short-term and long-term facilitation at the Drosophila neuromuscular junction. Proc Natl Acad Sci USA 75:515-519.

Jan YN, Jan LY, Dennis MJ (1977) Two mutations of synaptic transmission in Drosophila. Proc R Soc Lond [Biol] 198:87-108.

Kasbekar DP, Nelson JC, Hall LM (1987) Enhancer of seizure: a new genetic locus in Drosophila melanogaster defined by interactions with temperature-sensitive paralytic mutations. Genetics 116:423431.

Kernan MJ, Kuroda MI, Kreber R, Baker BS, Ganetzky B (1991) nap ${ }^{s,}$ a mutation affecting sodium channel activity in Drosophila, is an allele of $m l e$, a regulator of $\mathrm{X}$ chromosome transcription. Cell 66: 949-959.

King DG, Wyman RJ (1980) Anatomy of the giant fibre pathway in Drosophila. I. Three thoracic components of the pathway. J Neurocytol 9:753-770.

Koenig JH, Ikeda K (1983) Characterization of the intracellularly recorded response of identified flight motor neurons in Drosophila. $\mathbf{J}$ Comp Physiol 150:295-303.

Kosaka T, Ikeda K (1983) Possible temperature-dependent blockage of synaptic vesicle recycling induced by a single gene mutation in Drosophila. J Neurobiol 14:207-225.

Krishnan SN, Frei E, Swain GP, Wyman RJ (1993) Passover: a gene required for synaptic connectivity in the giant fiber system of Drosophila. Cell 73:967-977.

Levine JD, Hughes M (1973) Stereotaxic map of the muscle fibers in the indirect flight muscles of Drosophila melanogaster. J Morphol 140:153-158.

Loughney K, Kreber R, Ganetzky B (1989) Molecular analysis of the para locus, a sodium channel gene in Drosophila. Cell 58:11431154.

Mallart A, Angaut-Petit D, Bourret-Poulain C, Ferrus A (1991) Nerve terminal excitability and neuromuscular transmission in $\mathrm{T}(\mathrm{X} ; \mathrm{Y}) \mathrm{V7}$ and Shaker mutants of Drosophila melanogaster. J Neurogenet 7:7584.

Pavlidis P (1994) Physiological, behavioral and molecular analysis of bang-sensitive paralytic mutants of Drosophila melanogaster. Ph.D. Thesis, University of California, Berkeley.

Pavlidis P, Ramaswami M. Tanouye MA (1994) The Drosophila easily shocked gene: a mutation in a phospholipid synthetic pathway causes seizure, neuronal failure, and paralysis. Cell 79:23-33.

Royden CS (1988) The molecular analysis of $t k o$, a behavioral mutation in Drosophila melanogaster. PhD Thesis, University of California, San Francisco.

Royden CS, Pirrotta V, Jan LY (1987) The $t k o$ locus, site of a behavioral mutation in $D$. melanogaster, codes for a protein homologous to prokaryotic ribosomal protein S12. Cell 51:165-173.

Salkoff L, Kelly L (1976) Temperature-induced seizure and frequency- 
dependent neuromuscular block in a temperature sensitive mutant of Drosophila. Naturc 273:156-158.

Salkoff L, Wyman R (1980) Facilitation of membrane excitability in Drosophila. Proc Natl Acad Sci USA 77:6216-6220.

Schubiger M, Feng Y, Fambrough DM, Palka J (1994) A mutation of the Drosophila sodium pump $\alpha$ subunit results in bang-sensitive paralysis. Neuron 12:373-381.

Shoffner JM, Lott MT, Lezza AM, Seibel P, Ballinger SW, Wallace DC (1990) Myoclonic epilepsy and ragged-red fiber disease (MERRF) is associated with a mitochondrial DNA tRNA(Lys) mutation. Cell 61: 931-937.

Stern M, Ganetzky B (1989) Altered synaptic transmission in Drosophila Hyperkinetic mutants. J Neurogenet 5:215-228.
Tanouye MA, Wyman RJ (1980) Motor outputs of giant nerve fiber in Drosophila. J Neurophysiol 44:405-421.

Thomas JB, Wyman RJ (1984) Mutations altering synaptic connectivity between identified neurons in Drosophila. J Neurosci 4:530-538. van der Bliek AM, Meyerowitz EM (1991) Dynamin-like protein encoded by the Drosophila shibire gene associated with vesicular traffic. Nature 351:411-414.

Wu C-F, Ganetzky B (1980) Genetic alteration of nerve membrane excitability in temperature-sensitive paralytic mutants of Drosophila melanogaster. Nature 286:814-816.

Wu C-F, Ganetzky B (1992) Neurogenetic studies of ion channels in Drosophila. In: Ion channels (Narahashi T, ed), pp 261-314. New York: Plenum 